H俞MSA $\begin{aligned} & \text { Hamsa } \\ & \text { Journal of Judaic and Islamic Studies }\end{aligned}$

3 | 2017

Onomastics of Muslims and Jews

\title{
Nombres bíblicos: Pasado y presente
}

\section{Aaron Demsky}

\section{OpenEdition}

Journals

Edición electrónica

URL: https://journals.openedition.org/hamsa/638

DOI: 10.4000/hamsa.638

ISSN: 2183-2633

Editor

CIDEHUS - Centro Interdisciplinar de História Culturas e Sociedades da Universidade de Évora

Referencia electrónica

Aaron Demsky, «Nombres bíblicos: Pasado y presente», Hamsa [En línea], 3 | 2017, Publicado el 31 marzo 2017, consultado el 13 mayo 2021. URL: http://journals.openedition.org/hamsa/638 ; DOI: https://doi.org/10.4000/hamsa.638

\section{(9) $\odot \Theta \Theta$}

Hamsa est mise à disposition selon les termes de la Licence Creative Commons Attribution - Pas d'Utilisation Commerciale - Pas de Modification 4.0 International. 


\title{
Nombres bíblicos: Pasado y presente
}

\section{Aaron Demsky}

Bar-Ilan University

\section{Resumo:}

O campo da onomástica judaica é multifacetado, cobrindo um período de mais de 3000 anos, em qua a história judaica interage com as culturas que a rodeiam. Neste artigo, procede-se a uma análise, em tempo longo, dos nomes próprios, sobretudo os que se encontram na Bíblia, num amplo recorrido até ao Israel atual.

Palavras-chave: Nomes próprios, nomes bíblicos, onomástica judaica

\begin{abstract}
:
The field of Jewish onomastics is multifaceted covering a period of over 3000 years of Jewish history interacting with the surrounding cultures. This historical overview intends a long-term analysis of proper names, especially those found in the Bible, from the past to present-day Israel.
\end{abstract}

Keywords: names, biblical names, Jewish onomastics

\section{Visión de conjunto}

La Biblia Hebrea es el texto fundamental para las religiones monoteístas y proporciona un conjunto de unos seiscientos nombres personales, algunos de los cuales han sido adoptados por judíos, cristianos y musulmanes. Los nombres hebreos de origen bíblico se complementan también con datos epigráficos, especialmente los proporcionados por sellos personales, además del Papiro de Elefantina y tablillas de arcilla babilónicas. Los nombres hebreos forman parte de una de las ramas del antiguo sistema onomástico de las lenguas semíticas, que se documenta tanto en acadio, antiguo sudarábico, ugarítico como en fuentes cananeas y arameas.

Las asociaciones positivas, la autoridad religiosa y la belleza literaria de los relatos bíblicos han permitido la creación de muchos nombres hebreos en sus formas locales vernáculas, popularizándose ampliamente en la cultura occidental. Así se refleja, por ejemplo, en la aceptación que en los Estados Unidos han tenido los nombres masculinos como Jacob, David y Mateo o los nombres femeninos de Sara, Abigail y Hannah.

Los nombres hebreos, como categorías onomásticas en general, son, además, de excepcional importancia para los estudios lingüísticos, la crítica literaria y, de manera especial, para la historia social, ya que permite la identificación de creencias populares y modas cambiantes que generalmente no se explicitan en fuentes escritas antiguas.

Los nombres hebreos pueden clasificarse en nombres proposicionales y epitéticos (descriptivos, abreviados). Los nombres proposicionales, tanto plenos como abreviados (hipocorísticos) son oraciones verbales o nominales en las que se sigue una estructura del tipo Sujeto + Predicado o Predicado + Sujeto, en la que el sujeto generalmente es una divinidad mencionada por su nombre, como, por ejemplo, YHWH (Yeho/Yahu/Ya/Yo), El, Baal, Shaddai, por títulos de autoridad (melekh, sar, 'adon, es decir, "rey", "príncipe", "señor", etc,), por 
términos de parentesco ('ab, 'em, 'aḥ, ham es decir, "padre", "tío/patrón", "hermano", "cuñado", respectivamente) o la forma arcaica șur "piedra, sílex" (Num. 1:5-19). "El es el nombre genérico para "Dios" y se encuentra profusamente documentado en el Antiguo Oriente. Ba'al ("señor") es más ambiguo, ya que es el apelativo sirio-cananeo de la divinidad de la tormenta, Hadad. ${ }^{1}$ En épocas tempranas, el término $b a^{\prime} a l$ fue usado por los israelitas para designar a YHWH, así Meribb'al, Ishba'al (descendente del rey Saúl), el hijo del rey David, Ba'alyah (1 Cro. 12: 5) y en el Reino del Norte, como se evidencia en el Ostraca de Samaría, a diferencia de Oseas $(2,18-19)$. Los nombres israelitas derivados de $B a^{\prime} a l$ que se encuentran en los primeros profetas (pero no en la versión paralela de Crónicas) fueron con posterioridad "corregidos" sustituyendo la forma peyorativa boshet (vergüenza) por el elemento teóforo $B a^{\prime} a l$, leyéndose así Mephiboshet o Ish-Boshet.

El verbo se presenta bajo la forma de perfectivo qâtal (Netanel o Elnatan - 'Dios (EI) dio') o bajo la forma de imperfectivo yiqtal (Yekhonyahu or Yehoyakhin - 'Que YHWH establezca'). Ambos tipos nominales y verbales pueden abreviarse eliminando el sujeto (Yehonatan , Nathan) o el predicado (Eliab , Eli) como forma hipocorística. En ocasiones, la forma apocopada se conserva con vocal larga (Azaryahu > Ezra).

Esta tipología ayuda a valorar la veracidad de los relatos bíblicos así como los desarrollos teológicos y las distintas modas en torno a la imposición del nombre a lo largo de un periodo de miles de años. Así, por ejemplo, en la denominada época patriarcal, aproximadamente hacia mediados y finales del segundo milenio a.C, se atestigua que la forma yiqtal + El era popular tanto en las narraciones bíblicas como en las designaciones coetáneas amoritas (cananeas): Yishma+el (Ishmael), Yisra+el (Israel), con frecuencia sin la marca de divinidad El, Ya'aqov (Jacob), Yosef (Joseph), Yitzhaq (Isaac), lo que daría credibilidad al temprano desarrollo de estas tradiciones bíblicas.

Los nombres extranjeros se introdujeron en los distintos periodos de exilio y solo fueron considerados nombres hebreos en épocas posteriores: la estancia en Egipto dio lugar a los nombres Moisés, Aaron, Phineas, Hur y Miriam. En el último exilio persa-babilónico (desde 586 a.C. en adelante), se introdujeron nombres vernáculos como Zerubabel, Mordejai, Ester y Ciro.

\section{El nombre divino YHWH}

Un desarrollo teológico importante en la constitución del monoteísmo israelita fue la aparición del nombre divino inefable $\mathrm{YHWH}$, es decir, el del nombre personal $\mathrm{y}$ autoproclamado de la Divinidad: "Yo soy YHWH. Me aparecí a Abraham, a Isaac y a Jacob como "El-Shadday, pero por mi nombre de YHWH no me di a conocer a ellos" (Ex. 6: 2,3). La pronunciación del tetragrammaton se ha malinterpretado en la literatura académica moderna así como en diversos círculos cristianos; entre los primeros, interpretando Yahveh como una forma causativa; entre los segundos, leyendo erróneamente Jehovah. Significativamente fue Moisés al que se le considera haber creado el primer nombre personal israelita introduciendo este elemento teofórico al renombrar a su protegido Yehoshua (Num. 13:16). Esta denominación de la divinidad nacional con varias formas abreviadas ( $Y w, Y e h w$, como en el nombre personal Yehonatan/Jonatan, como primer elemento, o Yahu/Yah, como segundo elemento, así en Yeshayahu/Isaías) se popularizó en Israel durante el periodo monárquico (1000-586 a. C), tal y como se evidencia a través del creciente número de personalidades que incluyeron este elemento teóforo en los libros proféticos.

Además, los testimonios epigráficos de la Edad de Hierro, sin haber sido editados, muestran que este elemento divino fue el más popular en círculos israelitas y de Judea. Sorprendentemente, la escasez de otras divinidades en fuentes onomásticas extrabíblicas

\footnotetext{
${ }^{1}$ También se encuentra en el apodo babilónico Bel referido a la Divinidad más importante, Marduk, vid. Jes. 46,1;Jer. 50,2;51:44.
} 
muestra que los primeros israelitas tuvieron una gran fidelidad a la forma $\mathrm{YHWH}^{2}$ a través de la cual reconocían su carácter benefactor, protector y justo, tal y como se refleja en el uso de estas formas onomásticas. ${ }^{3}$

Algunos nombres femeninos tienen un elemento divino, como Elisheba ("Mi Dios" - como juramento), Bitiah ("hija de $\mathrm{YHWH}^{\prime}$ ), o la forma sustitutiva de parentesco Ahinoam ("Mi hermano (divino) es agradable"); sin embargo, muchos son nombres de animales: Rebeca ("ternero engordado"), Leah ("vaca") y Raquel ("oveja"), mostrando la gran importancia que el ganado bovino tuvo en estas sociedades patriarcales pastoriles. La mujer de Moisés era Ziporah ("pájaro"). Otras dos mujeres destacadas de la época de los Jueces fueron Yael ("gacela") y Débora ("abeja").

\section{La evidencia epigráfica}

Otra fuente para el estudio de los nombres hebreos bíblicos es la epigrafía hebrea. A través de ella, se pueden documentar cientos de nombres distintos de ese periodo escritos en ostraca o piedra, especialmente inscritos en sellos personales. ${ }^{4}$

Estos nombres no han sido editados ni se han modificado desde la época bíblica en la que fueron usados o escritos. Como he mencionado anteriormente en relación a la alta frecuencia con la que los nombres teofóros yahvistas fueron usados para expresar creencias religiosas, puede aprenderse mucho sobre las modas populares a la hora de elegir nombres e incluso de algunas prácticas administrativas del momento.

Así, por ejemplo, los sellos o bullae que incluyen nombres de mujeres pueden dividirse entre aquellos en los que la mujer es identificada con el nombre de su padre, como bat ("hija de") o el nombre de su marido, bien con la forma 'eshet ("mujer de") o la más honorífica de 'amat ("criada de")..$^{5}$ Tal y como menciona el profeta Isaías, las mujeres querían ser identificadas adecuadamente en esa sociedad patriarcal en relación con su marido, como se alude en Is. 4, 1: "Siete mujeres se agarrarán a un solo hombre en aquel día, diciendo: 'Nuestro pan comeremos y nuestro manto vestiremos, con tal de que seamos denominadas con tu nombre; iquítanos el oprobio!'."

Imagen 1. Sello personal, perteneciente a 'Immadiyahu, hija de Shubanyahu' לעמדיהו בת שבניהו

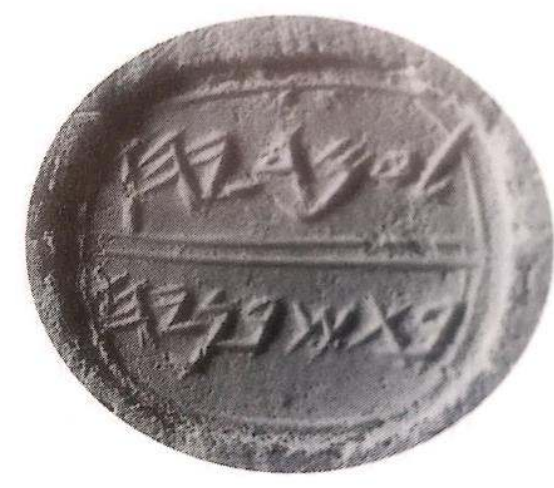

2 J. H. Tigay, You Shall Have No Other Gods- Israelite Religion in the Light of Hebrew Inscriptions, Atlanta, Scholars Press, 1986.

${ }^{3}$ Mitka Golub, "The Distribution of Personal Names in the Land of Israel and Transjordan during the Iron II Period", Journal of the American Oriental Society 134/4 (2014), p. 621-642.

${ }^{4}$ N. Avigad and B. Sass, Corpus of West Semitic Stamp Seals, Jerusalem, Israel Exploration Society, 1997; Mitka Golub, "The Distribution of Personal Names...".

${ }^{5}$ N. Avigad and B. Sass, Corpus of West Semitic Stamp Seals..., p. 63-65. 
Otra característica del material epigráfico es la identificación de tres generaciones mediante el nombre patronímico y del abuelo. Esta forma temprana de trinomia se encuentra en sellos personales desde la época final de la monarquía del reino de Judá (siglo $\mathrm{VI}$ a.C) y se designa en hebreo con el término meshulashim. Este uso administrativo fue adoptado por Jeremías con fines literarios para crear un subtexto en su discurso a través de la comparación de distintos individuos que comparten el mismo abuelo: Baruch ben Neriah ben Mahseiah y su hermano Seraiah (Jer. 32:12; 51:59), o Gedaliah ben Ahiqam ben Shaphan, miembro de una ilustre familia de oficiales reales (Jer. 39:14; 45:5,9, 26:24, 36:11 y cf. 2Re 22:3).

Esta misma forma de designación onomástica se encuentra también en sociedades modernas en las que existe un conjunto limitado de nombres con estas características con el fin de poder identificar de forma mucho más precisa a los individuos. Además de incluir el nombre de familia y un metrónimo, otra de las formas más básicas, como en algunas sociedades árabes, es la de enumerar: 'ism al shahsi (nombre personal), 'ism al 'ab (patronímico) y 'ism al jed (nombre del abuelo).

\section{La función literaria en los nombres bíblicos}

En la Biblia se encuentran muchas referencias a los nombres y a sus significados. Por ejemplo, Abigail, hablando sobre su marido Nabal, hace un juego de palabras con su nombre: "כי כשמו כן הוא ,Pues su nombre quiere decir el loco y se ha dejado llevar por la locura" (I Sam, 25:25). En realidad, Abigail está expresando con sus propias palabras la idea universal de nomen est omen o de la expresión árabe ismo ŷismo, es decir, la creencia de que el nombre personal refleja los rasgos de personalidad de aquel que es nombrado. Una idea similar se encuentra en Ecl. 7:1, en donde, a través de un nuevo juego de palabras, se identifica el nombre personal con la reputación del nominado: "Un buen nombre (shem) es mejor que aceite perfumado (shemen).

El libro de Rut, que está lleno de referencias onomásticas, es un notable ejemplo de la importancia de la función literaria de los nombres en el texto bíblico. ${ }^{6} \mathrm{El}$ autor de este libro tan popular muestra una especial sensibilidad a la hora de expresar la personalidad de sus personajes a través del juego simbólico que realiza con sus nombres personales. Así, por ejemplo, confronta el nombre de Naomi, "agrado", un significado que no se corresponde ya con su situación personal, con el de Mara, "amargura", nombre personal con el que vuelve a nombrarla en Rut 1:20. Desde el comienzo del relato se utiliza la ironía al hablar de la época de hambruna que asolaba el lugar de Bethlehem, literalmente "La casa de pan (o alimento)", 7 y contrastándolo después con el desconocido destino al que tuvo que desplazarse "a los campos de Moab". A este último topónimo lo considero un "innominado", que es utilizado como un recurso literario para expresar la opinión crítica del autor. Otro uso de estos nombres "innominados" es el del pariente varón directo que debe redimir (goel) y asumir la responsabilidad del cuidado y sustento de la empobrecida viuda Naomi y su hijastra Rut. A este se le denomina peyorativamente Peloni Almoni (Rut. 4:1), puesto que él no cumplirá su deber. ${ }^{8}$

Otro procedimiento de esta función literaria es el uso de nombres de hermanos como el caso de Mahlon y Kilion (Rut. 1:5). Estos nombres generalmente comparten un mismo significado o formas morfológicas relacionadas. ${ }^{9}$ Asimismo, un nuevo juego de palabras se

\footnotetext{
${ }^{6}$ Aaron Demsky, "Names and No-Names in the Book of Ruth", in These Are the Names- Studies in Jewish Onomastics, éd. A. Demsky, J.A. Reif, J. Tabory, Ramat Gan, Bar-Ilan University Press, Vol. 1, p 27-37.

7 En árabe, "Casa de carne" (lahm).

${ }^{8}$ Cf. Deut 25:10; vid. también el nombre Palmoni (Dan 8:13). La forma árabe relacionada, fulân, que se refiere a aquella persona de poca importancia, ha dado lugar en español a "Señor Fulano".

${ }^{9}$ Estos nombres de parentesco entre hermanos, como Eldad y Medad (Num. 11:26) o Peresh y Sheresh (I Cron. 7:16), no se encuentran tan solo en la Biblia, sino en otros muchos textos, como, por ejemplo, la
} 
encuentra en Rut 2:20, en donde el nombre de Boaz (cuyas radicales hebreas son beth, 'ain y zain) es relacionado por Naomí con la forma verbal 'azb (mediante metátesis de las mismas radicales hebreas 'ain, zain y bet), para expresar la misericordia de Dios por no haber abandonado ni a los vivos ni a los muertos, un rasgo de profunda misericordia que posee Boaz. En definitiva, con estos recursos onomásticos, el autor ha sabido convertir una tradición histórica sobre los antepasados del rey David en una historia llena de humanidad que ya se considera un relato de significado universal.

Otro aspecto destacado de usos onomásticos de los nombres bíblicos es lo que se denomina en hebreo midrash shem, es decir, el conjunto de explicaciones que se ofrecen en un texto para comprender el significado de nombres especialmente importantes ${ }^{10}$. Estos nombres pueden explicarse así a través de juegos de palabras, falsas etimologías o adivinanzas encubiertas ( $c f$. el caso de Boaz aludido con anterioridad). Estos procedimientos tienen una función literaria y estética a la hora de impulsar el hilo de la historia y enfatizar su mensaje. Por supuesto que las explicaciones de estos nombres están muy lejos de poder considerarse etimologías rigurosas desde el punto de vista lingüistico que puedan explicarse a través de la derivación u otros procedimientos morfológicos o semánticos. De algún modo, esta forma de atribuir un sentido asociativo a los nombres que se otorgan a los recién nacidos debe relacionarse más bien con los deseos de los propios padres así como a la idea de que su significado determinará el carácter o la esencia de la persona nombrada.

Dos últimos ejemplos pueden servir para ilustrar esto. Cuando la hija del Faraón encuentra al recién nacido israelita entre los juncos, esta le otorga el nombre adoptivo de Moisés. Es muy probable que el término derive del egipcio mes, "niño"; sin embargo, el texto bíblico lo explica a través de la expresión hebrea כי מן המים משיתיהו "Lo he sacado (meshitihu) de las aguas" (Ex. 2:10). Tras la lucha de Jacob con el ángel, a aquel se le otorga un nombre adicional más adecuado, como el de Israel, que significa literalmente "Dios gobierna". Sin embargo, en el texto el nombre se explica como un recurso para manifestar el carácter del propio Jacob: "En adelante ya no te llamarás Jacob, sino Israel, porque has luchado con Dios y con los hombres y has salido vencedor" (Gen. 32:29).

\section{5. Época del Segundo Templo (538 a.C - 70 d.C)}

Durante el periodo del Segundo Templo, los nombres hebreos reflejan los efectos de procesos históricos decisivos, tales como la desaparición de las primitivas sociedades tribales, la creación de la Diáspora judía y la subsiguiente influencia e impacto del arameo y del griego ${ }^{11}$ en las designaciones onomásticas. Esto trajo consigo la adopción de nombres de origen no hebreo así como la costumbre de utilizar dos nombres, uno hebreo y otro vernáculo. ${ }^{12}$ Por otro lado, la introducción de la costumbre popular de la paponomía, esto es, el nombrar a través de

alusión árabe a los enemigos bíblicos Harun Qarun (Aaron y Korah, basado en el Corán, Sura 28) y, por supuesto, en los conocidos hermanos Hansel y Gretel, diminutivos de Johannes y Margareta.

${ }^{10}$ Yair Zakovitch, "Explicit and Implicit Name-derivations", Hebrew Annual Review 4 (1980), p. 167-181; Moshe Garsiel, Biblical names - A Literary Study of Midrashic Derivations and Puns, Ramat-Gan, Bar Ilan University Press, 1991.

${ }^{11}$ Tal Ilan, Lexicon of Jewish Names in Late Antiquity, Tuebingen, Mohr Siebeck, Part I Palestine 330BCE200CE (2002); Part II Palestine 200-650 (2012); Part III The Western Diaspora 330 BCE-650 CE (2008); Part IV The Eastern Diaspora 330 BCE-650CE (2011); M. Cassuto-Salzmann "Greek Names among the Jews", Eretz-Israel 3 (1954), p. 186-190.

12 Aaron Demsky, "Double Names in the Babylonian Exile and the Identity of Sheshbazzar", in These Are the Names: Studies in Jewish Onomastics, éd. A. Demsky, Vol. 2, Ramat Gan, Bar Ilan University Press, 1999, p. 23-40. 
la filiación de la generación de los abuelos, redujo el número de los nombres más creativos y singulares del periodo bíblico más temprano. ${ }^{13}$

Otro aspecto decisivo de este periodo fue la canonización del texto bíblico y la fijación de la mejor lectura del texto sagrado, lo que provocó la creación de un notable número de nuevos nombres hebreos. Algunos eran completamente originales, pero otros surgieron como consecuencia de una mala lectura o una mala interpretación de extranjerismos o términos técnicos, especialmente aquellos relacionados con formas de parentesco. Aún más, muchos de estos nombres fueron el resultado de interpretaciones intencionadas surgidas a la luz de una nueva sensibilidad. En cualquier caso, una vez que el texto bíblico fue canonizado, todos estos nombres fueron considerados como legítimas formas onomásticas de origen hebreo.

A este tipo de nombres los he denominado "nombres fantasmas" (en hebreo, shem refa'im), una categoría onomástica que no ha sido adecuadamente reconocida en los estudios onomásticos. ${ }^{14}$ Los "nombres fantasma" son, en su mayoría, formas accidentales que no deben confundirse con otras categorías, como los "innominados", a los que se ha aludido con anterioridad. No obstante, todos estos desarrollos onomásticos han enriquecido y potenciado la reserva de nombres judíos que se ha ampliado de forma continuada desde la Antigüedad. ${ }^{15}$

Aunque muchos de los "nombres-fantasma" surgieron como consecuencia de las dificultades que presentaba el texto bíblico, también es posible considerar que un determinado nombre es el resultado de una interpretación tardía en la que se sugiere un nuevo significado a un determinado pasaje, como es el caso, por ejemplo, de las genealogías tribales (I Cron. 1-9). Estas genealogías, que reflejan los modelos de parentesco tribal en un contexto social muy temprano, perdieron su relevancia histórica ya en la época del Segundo Templo, por lo que fue esa nueva aproximación exegética la que en último término llevó a la invención intencional del nuevo nombre.

Un ejemplo de ello puede ser el caso del nombre hebreo tardío Avigdor, heb. Avi-Gedor: "Yered, el (clan) padre de (el lugar de) Gedor" (1Cr. 4,18). En un intento por ofrecer un significado nuevo y relevante a estas genealogías tribales primitivas y su terminología, la exégesis rabínica identificó el nuevo nombre Avigdor con Moisés (TB, Megillah 13a). Esta innovación es especialmente notable ya que anula el hecho de que esta genealogía de $1 \mathrm{Cr}$. 4,18 se refiere al clan de Judá y no al de Leví, la tribu de Moisés. Además, cambia el significado de Avi, que pasa a significar aquí "clan familiar/jefe" del lugar de Gedor. De este modo, esta nueva formación de Avigdor puede interpretarse como una nueva forma de definir la identidad colectiva de Israel que pasó de las primitivas alianzas tribales al modelo supra-tribal y nacional que desarrolló el judaísmo en la época del Segundo Templo.

\section{Alta Edad Media}

Una de las características más destacadas de este periodo, ya desde el s. VIII d.C, es la revitalización de muchos nombres bíblicos referidos a personajes preeminentes (tales como Abraham, Moisés y David) que, sin embargo, habían quedado relegados al silencio en épocas anteriores. Es posible que estos nuevos usos se debieran a la popularidad de algunos nombres como Ibrahim, Musa y Daud, así como otros personajes bíblicos, que son citados en el Corán y que se extendieron en sociedades arabe hablantes con población judía. Estos nombres, debido

\footnotetext{
${ }^{13}$ Aaron Demsky, "New Hebrew Names in the Second Temple Period: A By-product of Biblical Exegesis", in Names and Their Environment, Vol. 3. Anthroponomastics, Proceedings of the $25^{\text {th }}$ International Congress of Onomastic Sciences 24, éd. Carole Hough and Daria Izdebska, Glasgow, University of Glasgow, 2016, p. 41-51.

${ }^{14}$ Aaron Demsky, "Ghost Names" in the Bible", in in These Are the Names: Studies in Jewish Onomastics, éd. A. Demsky, Vol. 5, Ramat Gan, Bar Ilan University Press, 2011, p. 119-129.

${ }^{15}$ Tal Ilan, Lexicon of Jewish Names in Late Antiquity...
} 
al influjo cultural de ambas religiones, fueron incorporados también al sistema onomástico judío.

\section{Europa oriental}

En estos territorios los nombres bíblicos también tuvieron una gran importancia en la forja de la identidad judía. Así, ya en el s. XVI, en la Europa del Este, entre la población judía de origen asquenazí, se encuentra ampliamente extendido el fenómeno de los nombres dobles, uno en hebreo, y el otro en la lengua vernácula de la comunidad, el yiddish. En realidad, el nombre en Yiddish funciona como apelativo (heb. kinnui) del nombre hebreo. En ocasiones, se traduce Yitzhak - Isak; otros, son diminutivos como Yaakov (Jacob) - Kopel. Estos nombres dobles están relacionados entre sí y pueden tener variantes fácilmente reconocibles. Especialmente interesantes son aquellos nombres dobles que se han formado por ser palabras consecutivas procedentes del texto bíblico, en particular, de las Bendiciones de Jacob a sus hijos (Gen. 49:1-27): Binyamin Zeev ("lobo"), Yehudah (Judha) Aryeh (Leib), o variantes como Issachar Dov ("oso") o Naftalí Zvi (Hirsch) o los nombres femeninos como Hayah Sarah o Esther Malkah. Consideramos que estas formas onomásticas de estos nombres judíos son el resultado de la influencia de los libros impresos, especialmente la Biblia, que pudieran encontrarse en las casas y muestran el desarrollo del alfabetismo entre un gran número de hombres y mujeres que forjaron nuevas modas e innovaciones a la hora de elegir el nombre de sus hijos.

\section{Holocausto}

Con la llegada al poder de los nazis en Alemania en la década de 1930, el gobierno impulsó su política racial de aislar y perseguir a los ciudadanos judíos. Al principio, con la demarcación de negocios y productos judíos, a la que siguió, en agosto de 1938, la promulgación del abominable decreto por el que se obligaba a todos los judíos a incluir un nombre hebreo a sus documentos oficiales. Estos debían ser Israel para los hombres y Sara para las mujeres. Este modo de estigmatización de la población judía pronto fue adoptado por el gobierno austriaco y seguido, en 1940, por el régimen búlgaro. ${ }^{16}$ Irónicamente, estos dos nombres comparten la misma raíz, con el significado de "superar y gobernar".

\footnotetext{
${ }^{16}$ Saul Friedlander, Nazi Germany and the Jews, London, Harper Perennial, 1997, vol. 1, p. 254-255.
} 
Imagen 2

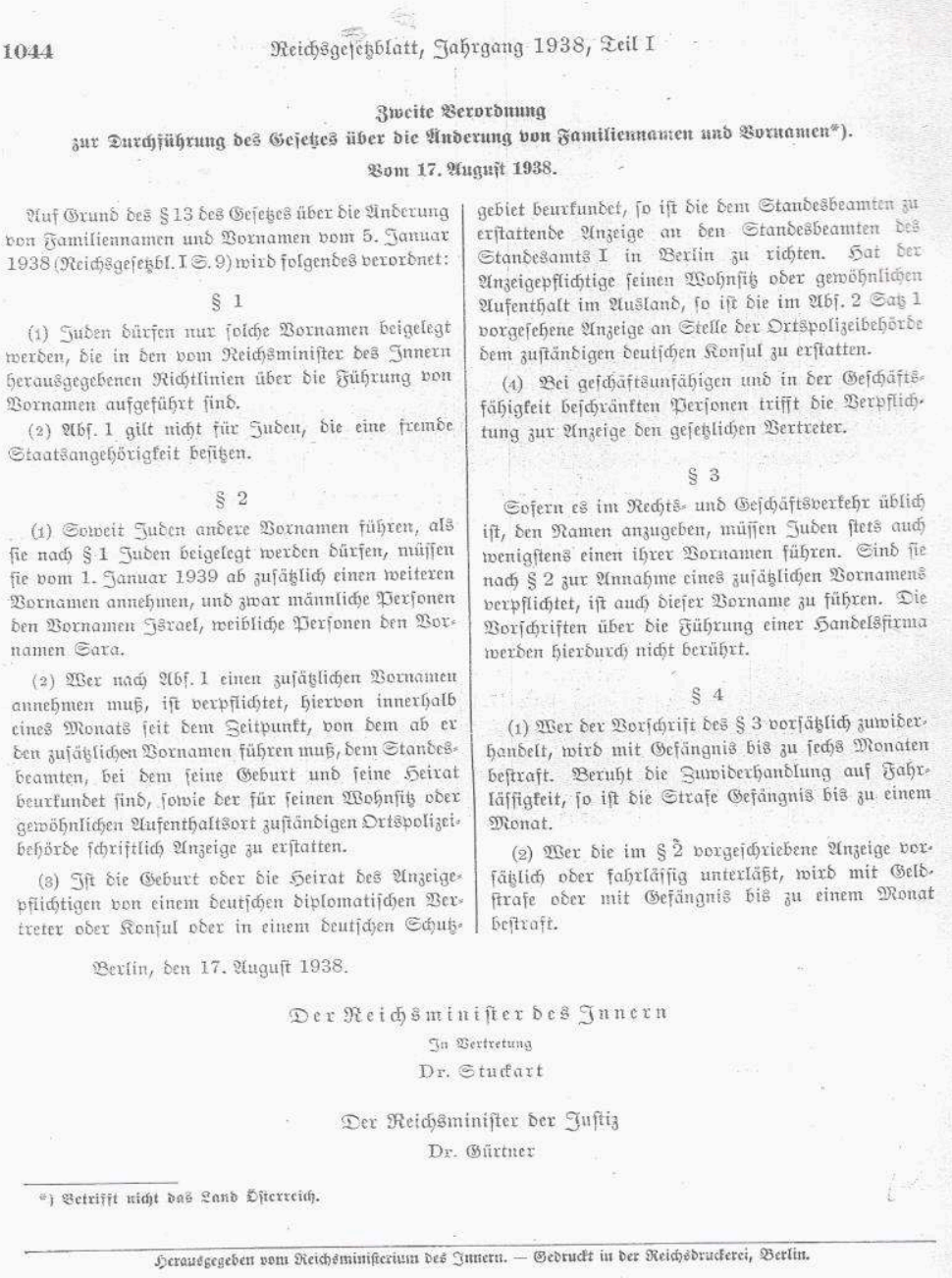

\section{Israel moderno}

Los judíos que llegaron a Israel en los siglos XIX y XX contribuyendo a su renacimiento nacional han enriquecido también la onomástica judía. La revitalización de la lengua hebrea se inició en el siglo XIX y fue acompañada por el resurgir de nombres bíblicos como Nehemías, Zerubabel y Ciro que recordaban el regreso a Sión de hace 2500 años.

Desde la década de 1920 en adelante, y como consecuencia de la llegada de un gran número de población judía secular, se produce la popularización de muchos nombres bíblicos que no formaban parte del tradicional elenco de nombres. ${ }^{17}$ En realidad, algunos de estas nuevas designaciones no habían triunfado con anterioridad porque hacían referencia a personajes bíblicos cuyos nombres tenían connotaciones negativas, como, por ejemplo, Nimrod, Hagar e incluso el de la Diosa cananea Anat. Al nombrar con estos nombres a sus hijos, esta nueva población judía consolidaría una tradición onomástica que rompía con la idea del exilio histórico y reafirmaba las vinculaciones bíblicas con la tierra. Así, nombres como

${ }^{17}$ Aharon Megged, "Names with Meanings" in in These Are the Names: Studies in Jewish Onomastics, éd. A. Demsky, vol 3, Ramat Gan, Bar Ilan University Press, 2002, p. 111-116 (en hebreo). 
Abner, Amotz, Yuval, Avishai, Asael, Yiftah (Jefta), Omri, Yigal, Yoram, Yael, Michal y Meirav se han convertido en nombres israelíes muy populares. Análogamente, ajustándose a la tendencia onomástica moderna de normalizar los géneros, algunos nombres masculinos desarrollaron formas femeninas propias, como, por ejemplo, Gabriela (Gabriel), Michaela (Michael), Daniela (Daniel), Ariela (Ariel) y Aharona (Aaron). Por otro lado, se otorgó también un nuevo significado al antiguo elemento 'am, "tío" (como en árabe) o "patrón" que se incluía en nombres bíblicos referentes a la Divinidad (como Amram) pasando a aludir de forma implícita al término hebreo 'am, "pueblo", "nación", en nombres como Yehiam o 'Amihai, "mi pueblo vive", en clara alusión al resurgir nacional del momento.

\section{Conclusión}

En este breve recorrido histórico sobre los nombres hebreos hemos tratado de mostrar el continuo diálogo que el pueblo judío ha establecido con sus raíces bíblicas a través de su sistema onomástico. Hemos analizado el rico y polifacético uso que se ha hecho de los nombres hebreos tanto con fines literarios como religiosos. También se ha analizado cómo estos nombres son un reflejo de los distintos periodos históricos que ha atravesado durante milenios, lo que ha permitido, finalmente, una mejor comprensión del influjo que la tradición onomástica judía ha podido ejercer en la cultura cristiana y musulmana. 